\title{
Natural Selection In Utero Contributes to the Male Longevity Deficit in Contemporary Human Populations
}

\author{
Ralph Catalano
}

University Of California, Berkeley 

Much literature invokes natural selection to explain the pervasive deficit in the average lifespan of men compared to women. ${ }^{1}$ The explanation assumes that mothers, not fathers, provisioned children over much of human existence, and that women who lived long enough to help their children and grand children survive to reproductive age had more grandchildren and great-grandchildren than did shorter-lived women. ${ }^{2}$ Although this argument implies that natural selection would conserve mutations that conferred longevity on mothers but not fathers, 3,4 it offers no explanation of the considerable changes over historic time in the male longevity deficit thereby implying that these arise solely from culture. 5 I show, however, that natural selection in utero empirically predicts variability over time in the deficit. This mechanism spontaneously aborts less fit fetuses during stressful times and reportedly selects more against males than females. My finding suggests that natural selection interacts with culture to predictably affect both the life span and sex ratio of contemporary human populations.

The fact that at least half, and as many as $70 \%$, of human conceptions end without live births makes gestation as much an opportunity for selection as for maturation. ${ }^{6}$ Theory suggests that natural selection has conserved mechanisms by which women spontaneously abort conceptuses and fetuses least likely to yield grandchildren. ${ }^{7}$ These mechanisms appear to select early in gestation against females that somehow signal that they or the ova of their potential offspring, which appear around the $6^{\text {th }}$ week of gestation, have chromosomal abnormalities. ${ }^{6}$ Later in gestation, the mechanisms supposedly select against fetuses, mostly small males, least likely to survive to reproductive age. 8 
Selection in utero assumes that mothers autonomically assess the fetal fitness and spontaneously abort those that fall below some criterion. ${ }^{9}$ Researchers have invoked this argument to explain clinically important phenomena such as the increase in birth defects among infants born to older women who may involuntarily risk lowering the criterion for abortion as opportunities to reproduce dwindle.10

More important for my purposes, the literature also uses selection in utero to explain more basic phenomena including variation over time in characteristics of populations. These include declines in the ratio of male to female births (i.e., the secondary sex ratio) in populations subjected to ambient stressors such as natural ${ }^{11}$ and human made disasters, 12 terrorist events, 13 extreme cold, 14 and economic contraction. 15 The explanation posits that women involuntarily manipulate the criterion for spontaneous abortion to avoid offspring that, if born, would least likely survive prevailing environmental conditions. ${ }^{9}$ More threatening environments raise the criterion thereby "culling" fetuses that mothers in more benign circumstances may have delivered live. 16

Males disproportionately populate the lower ranks of fetal fitness because sons, who require more of a mother's time and energy to sustain than do daughters, more likely die before reproducing than do females. A mother's investment in a son that died before reproducing would also deplete resources that she could have used to increase the reproductive chances of other live or yet-to-be-born children. So, when population stressors raise the "average" criterion for spontaneous abortion among pregnant women, male fetuses, given their relatively low fitness, disproportionately die thereby lowering the secondary sex ratio of their birth cohort. 16 
Research supports the argument that selection in utero culls birth cohorts of their least fit males. Men from low sex ratio, and presumably more culled, annual birth cohorts reportedly live longer than men from high sex ratio cohorts. $14,17,18$

The sex-ratio literature suggests that variability over time in the male longevity deficit may arise, at least in part, from selection in utero. Greater culling of male fetuses in stressful times implies that the difference between the lifespan of men and women should decline in low sex ratio birth cohorts; less culling of males in benign times implies that the difference in lifespan should increase in high sex ratio cohorts. I search for these patterns in data from Sweden for annual birth cohorts starting in 1751, the first year for which I can obtain data, and ending in 1916, the last in which sufficient mortality has occurred to estimate cohort lifespan. More specifically, I test the hypotheses that trends in the sex ratio of annual birth cohorts correlate positively with trends in the male longevity deficit, and that deviations from trends in the sex ratio of annual birth cohorts correlate positively with deviations from trends in the male longevity deficit.

I used data from Sweden because it has kept dependable vital statistics longer than any other nation-state. Combat deaths, moreover, distort estimates of Swedish lifespan less than those from other countries because Sweden has fought in relatively few wars.

I obtained vital statistics and lifespan data for Sweden from the Human Mortality Database.19 This source archives life table data only if they meet quality standards agreed among professional demographers and researchers. I used female lifespan, technically referred to as female cohort life expectancy at birth, less male lifespan as my 
measure of the male longevity deficit; and used the odds of a male birth as the secondary sex ratio.

I tested my hypotheses through the following steps. First, I used univariate BoxJenkins modeling to decompose the male longevity deficit and the secondary sex ratio into trends (i.e., values expected from autocorrelation including persistent increases or decreases, cycles, and oscillations) and annual deviations from trends. 20 । defined trend as the fitted values of the Box-Jenkins model, and annual deviations from trend as the residuals of the model. Second, I tested my hypothesized positive association between trends in the sex ratio and in the male longevity deficit by calculating the correlation between the fitted values of the two models estimated in step 1. Third, I tested my hypothesized positive association between annual deviations from trends in the sex ratio and male longevity deficit by calculating the correlation between the residuals of the two models. Results would support my hypotheses if the coefficients of correlation for both the sex-ratio variables significantly exceeded 0 .

The secondary sex ratio varied from 1.0211 in 1784 to 1.0703 in 1910 with a mean of 1.0490 over the 166 test years. The deficit in male lifespan ranged from 2.06 years for the cohort born in 1872 to 6.17 years for the 1916 cohort with a mean of 3.178 years. Figures 1 and 2 show the observed values of the sex ratio and of the male deficit as well as their trends estimated by Box-Jenkins modeling. Deviations from trend are the difference between the observed values and trend values.

Results from steps 2 and 3 supported the hypotheses in that the coefficient of correlation between trends in the male longevity deficit and secondary sex ratio was .27 $(\mathrm{SE}=.08)$ while that for annual deviations was $.40(\mathrm{SE}=.08)$. These coefficients imply 
that the sex ratio statistically "accounts" for about $7.5 \%$ of the variance in the trend in the male longevity deficit and about $16 \%$ of the variance in deviations from that trend. Consistent with my argument, these findings imply that cohorts culled in utero of their weakest males had the smallest male longevity deficits and vice versa.

To help give meaning to these correlations, I replicated my tests with regression equations that estimated time added or subtracted from the male deficit at different values of the sex ratio. The first equation estimated the relationship between trends while the second did so for annual deviations from trends. I applied the coefficient (i.e., 3.56) estimated in the equation for trends in the sex ratio to the minimum (1.0420), median (1.0492), and maximum (1.0563) values of the sex ratio trend variable. This exercise yielded results in which the male deficit was predicted to be 3.710 years at the minimum value of the trend in sex ratio, 3.736 years at the median value, and 3.761 years at the maximum value. As reported above, the range in the deficit over the test period was from 2.06 to 6.17 years.

The results for annual deviations of the sex ratio from its trend must be expressed as deviations of the male deficit from its trends. The median value of deviations of the sex ratio from its trend was, as expected, very close to 0 . I, therefore, calculated the deviation in the male deficit only for values at which the observed sex ratio was farthest below and above its trend. The deviation (i.e., -.0271) for the sex ratio most below trend predicts a value .343 years (or about 4 months) lower than expected from trend in the male longevity deficit. The deviation (i.e., .0193) for the sex ratio most above trend predicts a value .2449 years (or about 3 months) higher than expected from 
trend in the male deficit. Deviations from trend in the male deficit ranged from -.5193 years (or about - 6.2 months) to .5728 years (or 6.8 months).

The above estimations support the argument that natural selection may affect the male longevity deficit through selection in utero, a mechanism well-described in the theoretical and empirical literature, but heretofore not connected to the male longevity deficit. Replication would provide a sense of how widely in space and time my results may generalize. As noted, however, data from other societies describe shorter time periods and often reflect the influence of war on male longevity. These circumstances suggest that estimating the external validity of my findings will require more complex tests than mine. Keeping these tests accessible to a wide array of scholars may, therefore, prove challenging.

Human-made shocks, such as economic and political upheaval, may induce selection in utero implying that culture and conserved biological mechanisms interact to cause men to live less long than women. I, therefore, suggest that my findings add impetus for "consilience" between evolutionary and cultural perspectives on the pervasive and persistent male longevity deficit.

Methods

The best fitting Box-Jenkins model for the secondary sex ratio was:

$$
\frac{\mathrm{M}_{\mathrm{t}}}{\mathrm{F}_{\mathrm{t}}}=1.0493+\frac{1}{(1-.2149 \mathrm{~B})\left(1-.2430 \mathrm{~B}^{3}\right)} \mathrm{a}_{\mathrm{t}}
$$


$\mathrm{M}_{\mathrm{t}}$ is the number of live-born males in year $t$ and $\mathrm{F}_{t}$ is the number of live-born females, $a_{t}$ is the difference between the observed value at year $t$ and the value expected from the remainder of the equation, and $\mathrm{B}^{\mathrm{n}}$ is the backshift operator that implies its coefficient is applied to "a" at t-n. The 3 estimated values were at least twice their standard errors and the residuals of the model exhibited no autocorrelation.

The best fitting model for the longevity difference was:

$$
E_{f t}-E_{m t}=\frac{.2468 B}{\left(1-.2148 B^{6}\right)} a_{t}
$$

$E_{f t}$ and $E_{m t}$ are the cohort life expectancy (i.e., average age at death) for females and males born in Sweden in year t. The two estimated values were at least twice their standard errors and the residuals of the model exhibited no autocorrelation. 



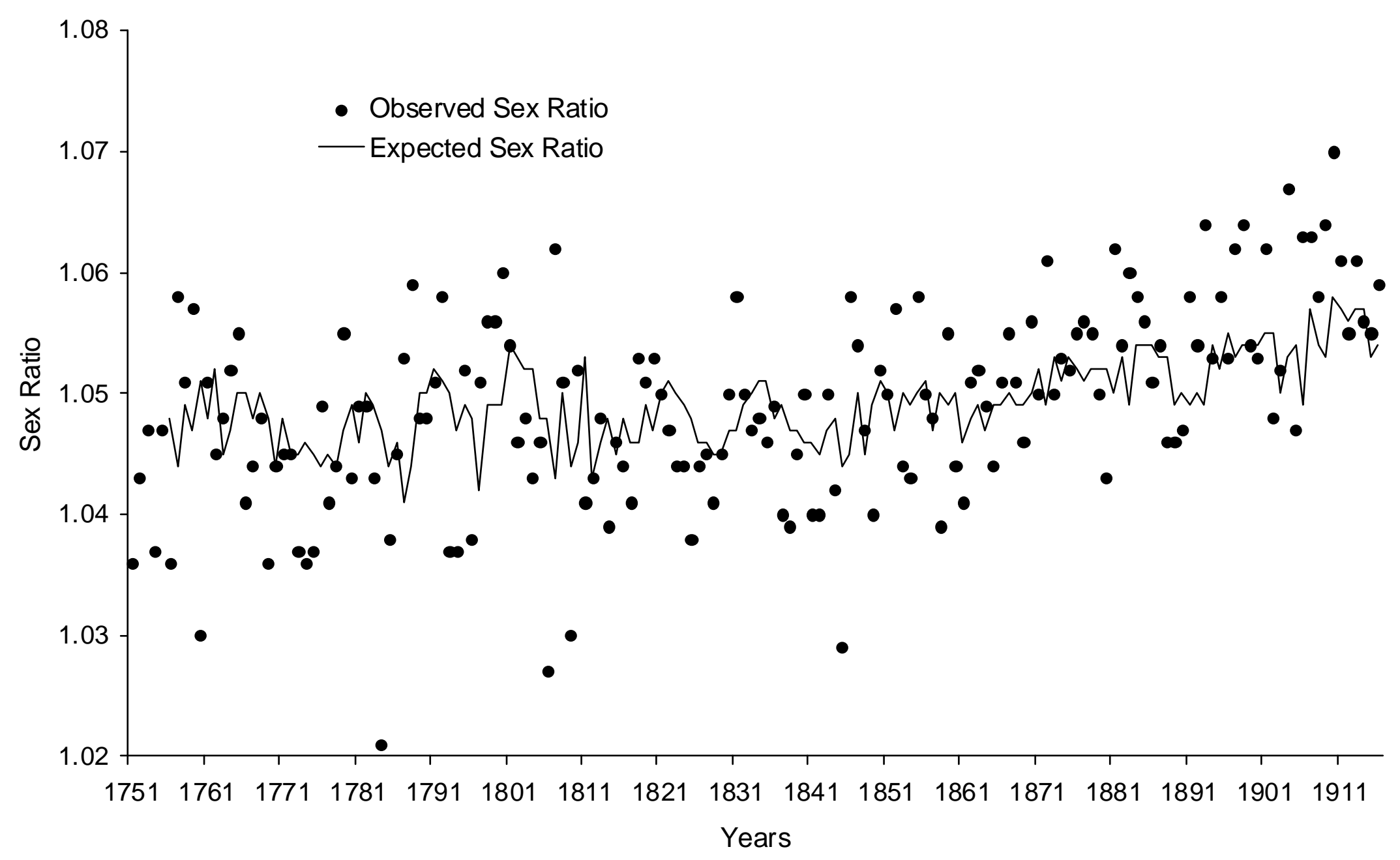


Figure 1. Observed and statistically expected values of the Swedish secondary sex ratio. First 5 years of expected values lost to modeling. 


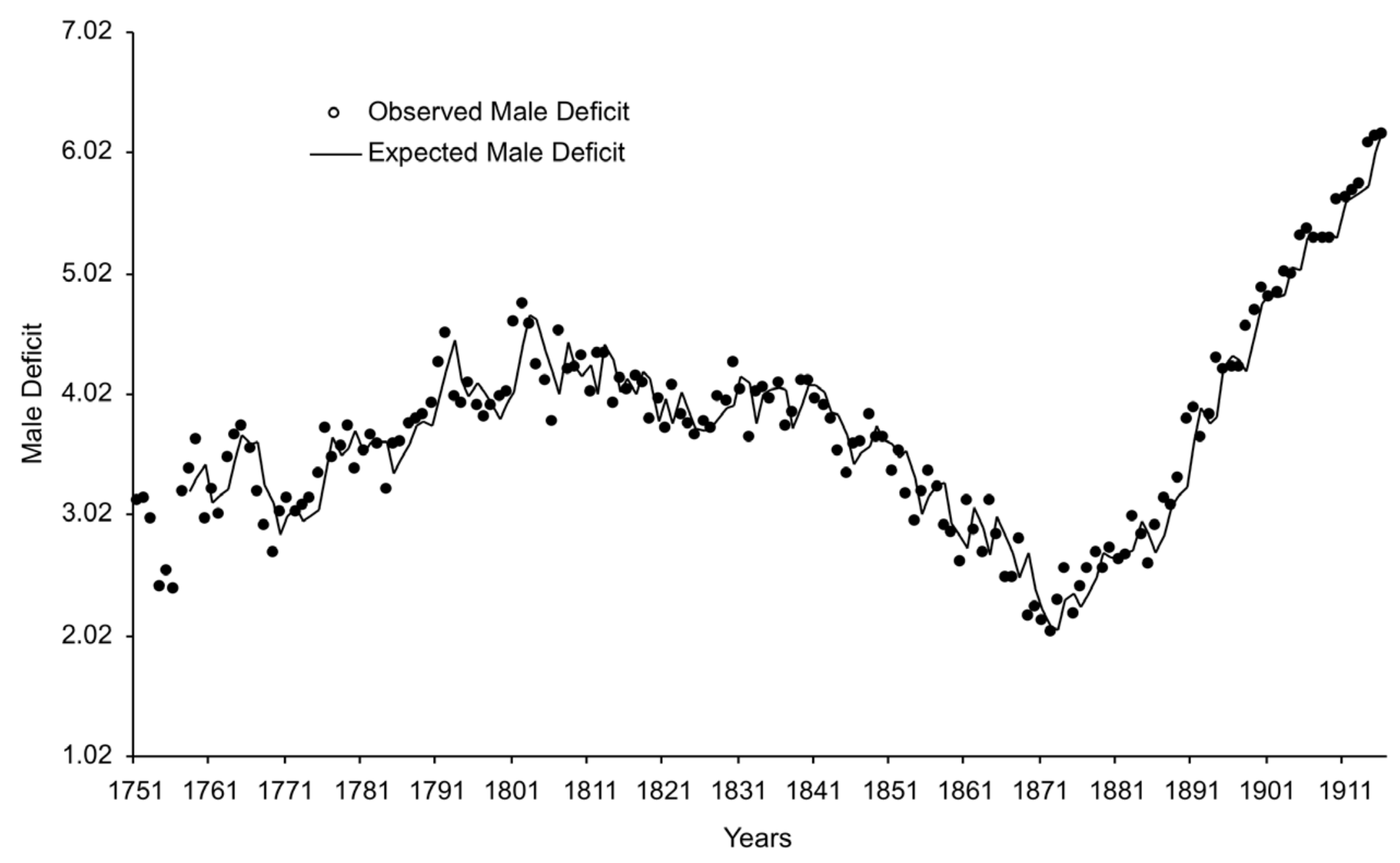


Figure 2. Observed and statistically expected values of the Swedish deficit in male lifespan. First 7 years of expected values lost to modeling. 
References

1. Peccei, J. A critique of the grandmother hypotheses: Old and new. Am. J. Hum. Biol. 13, 434-452 (2001)

2. O'Connell, J., Hawkes, N., \& Blurton Jones, J. Grandmothering and the evolution of Homo erectus. Hum. Evol. 36, 461-485 (1999)

3. Madrigal, L. \& Melendez-Obando, M. Grandmother's longevity negatively affects daughter's fertility. Am. J. Phys. Anthropol. 136, 223-229 (2008)

4. Shanley, D., Sear, R., Mace, R. \& Kirkwood, T. Testing evolutionary theories of menopause. Proc. R. Soc. B. 274, 2943-2949 (2007)

5. Moller, A., Fincher, C., \& Thornhill, R. Why men have shorter lives than women: Effects of resource availability, infectious disease, and senescence. Am. J. Hum. Biol. 21, 357-364 (2009)

6. Boklage, C. Survival probability of human conceptions from fertilization to term. Int. J. Fertil. 35, 75 (1990)

7. Forbes, L. The evolutionary biology of spontaneous abortions in humans. Trends Ecol. Evol. 12, 446-450 (1997).

8. Wells, J. Natural selection and sex differences in morbidity and mortality in early life. J. Theor. Biol. 202, 65-76 (2000)

9. Trivers, R., \& Willard, D. Natural selection of parental ability to vary the sex ratio of offspring. Science 179, 90-92 (1973) 
10. Neuhäuser, M., \& Krackow, S. Adaptive-filtering of trisomy 21: risk of Down Syndrome depends on family size and age of previous child. Naturwissenschaften. 94,117-121 (2007)

11. Fukuda, M., Fukuda, K., Shimizu, T., \& Moller, H. Decline in sex ratio at birth after Kobe earthquake. Hum. Reprod. 13, 2321-2322 (1998)

12. Lyster, W. Altered sex ratio after the London smog of 1952 and Brisbane flood of 1965. J. Obstet. Gynaecol. Br. Commonw. 81, 626-631 (1974)

13. Catalano, R., Bruckner, T., Marks, A. \& Eskenazi, B. Exogenouos shocks to the human sex ratio: the case of September 11, 2001 in New York City. Hum. Reprod. 21, 3127-3131 (2006)

14. Catalano, R., Bruckner, T., \& Smith, K. Ambient temperature predicts sex ratios and male longevity. Proc. Natl. Acad. Sci. U.S.A. 105, 2244-2247 (2008)

15. Catalano, R. Sex ratios in the two Germanies. Hum. Reprod. 18, 1972-1975 (2003)

16. Catalano, R. et al. A sex-specific test of selection in utero. J. Theor. Biol. 257, $475-479(2009)$

17. Catalano, R. \& Bruckner, T. Seconday sex ratios and male lifespan: damaged or culled cohorts. Proc. Natl. Acad. Sci. U.S.A. 103, 1639-1643 (2006)

18. Catalano, R. et al. Gender-specific selection in utero among contemporary human birth cohorts. Paed. Peri. Epi. 23, 273-278 (2009) 
19. Human Mortality Database. University of California, Berkeley (USA), and Max Planck Institute for Demographic Research (Germany). Available at www.mortality.org or www.humanmortality.de

20. Box, G., Jenkins, G. \& Reinsel, G. Time Series Analysis: Forecasting and Control (Prentice-Hall, London: Prentice-Hall, 1994) 


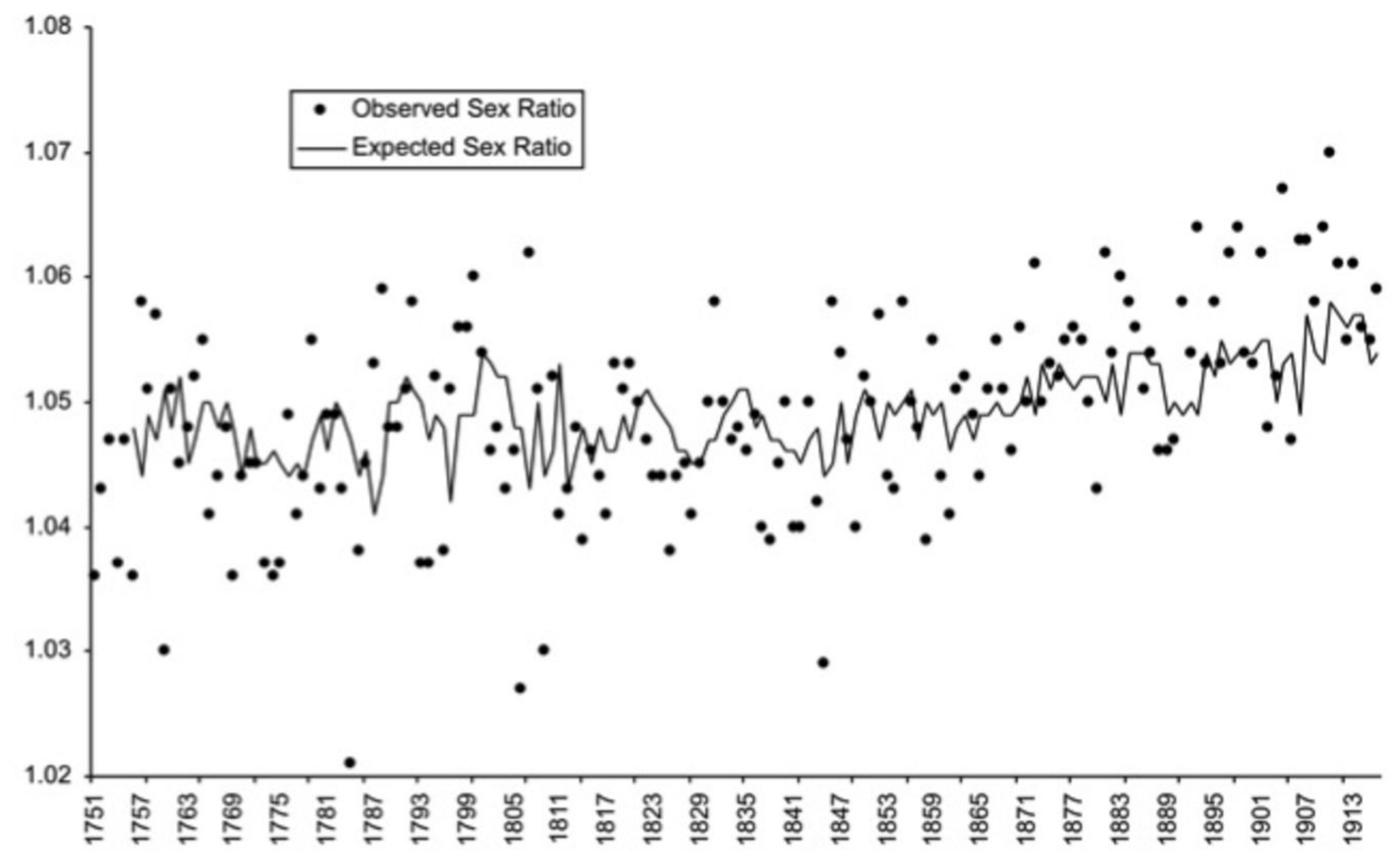




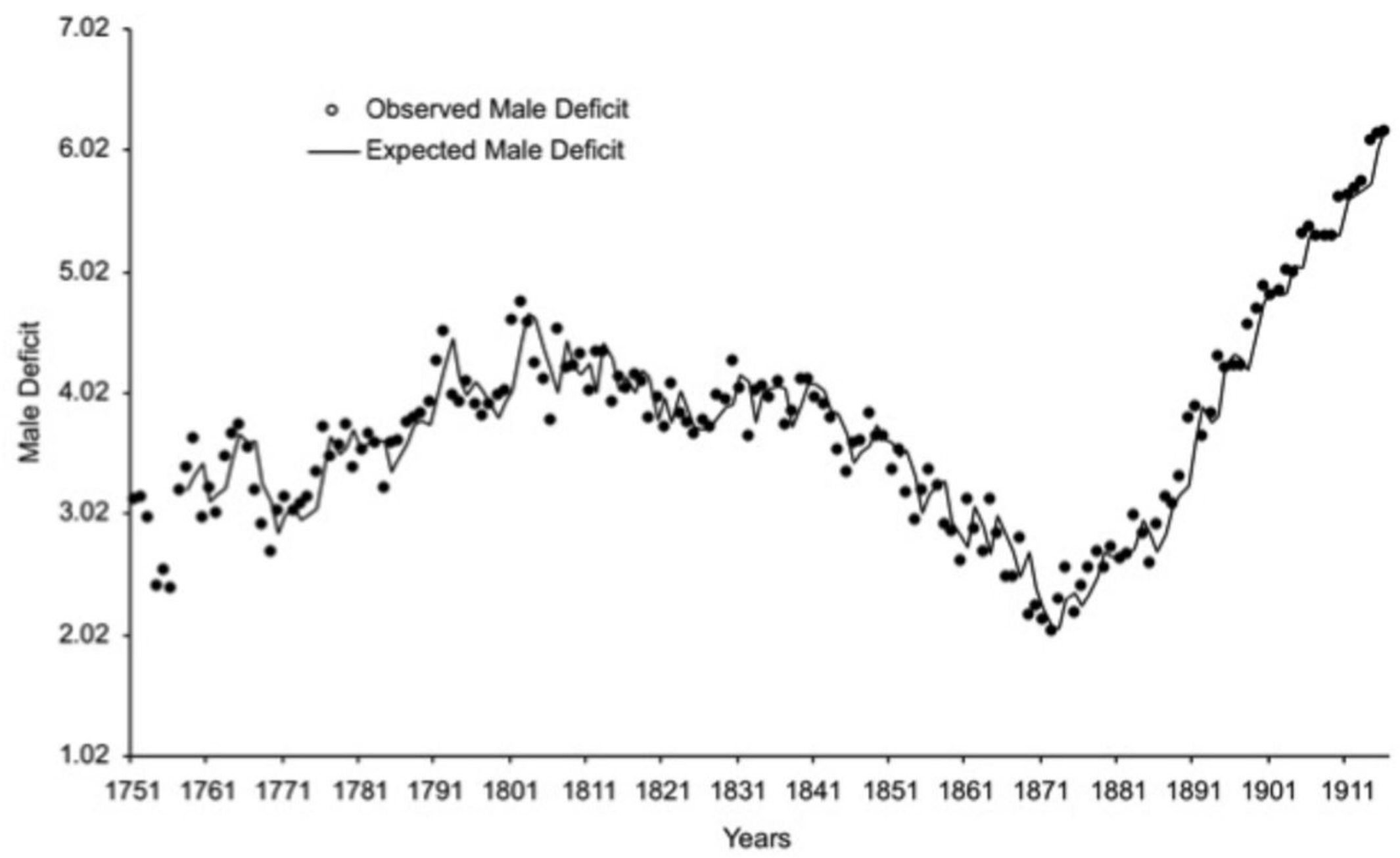

